\title{
Adjustment disorder considered
}

\section{ARTICLE}

\author{
Patricia Casey \& Faraz Jabbar
}

\begin{abstract}
SUMMARY
Adjustment disorder has been included in psychiatric classifications for over 40 years but has received little attention from the research community. It is particularly common in consultation liaison psychiatry. Evaluation is problematic since it may be mistaken for major depression, generalised anxiety or non-pathological reactions to stress. Its measurement by structured interview is difficult since it is not included in many instruments and, in others, cannot be diagnosed once the threshold for another disorder is reached. There are few evidence-based treatments and it is possible that these transient reactions may not require any formal intervention. Adjustment disorder generally carries an excellent prognosis but in some individuals is associated with selfharm and suicide.
\end{abstract}

\section{DECLARATION OF INTEREST}

P.C. is Editor of Advances in Psychiatric Treatment, but this article was commissioned and submitted before she took up this post. She has had no part in the refereeing of the manuscript or the decision to accept it for publication.

Adjustment disorder is not a new diagnostic category. It was incorporated into DSM-III in 1980 (American Psychiatric Association 1980) and into ICD-9 in 1978 (World Health Organization 1978), having been called transient situational disturbance in earlier editions. It represents a maladaptive but temporary reaction to life stressors. There are no detailed diagnostic criteria in either DSM-IV-TR (American Psychiatric Association 2000) or ICD-10 (World Health Organization 1992); the main clinical features described in those publications are summarised in Box 1.

Adjustment disorder differs from other diagnoses in which life events are a common factor. For example, an event or stressor is not essential for a diagnosis of depressive episode, whereas it is a prerequisite for a diagnosis of adjustment disorder. Apart from post-traumatic stress disorder (PTSD) and substance use disorder, adjustment disorder is the only other diagnosis based on aetiology in the current classifications. In ICD-10, adjustment disorder is classified under 'Neurotic, stressrelated and somatoform disorders' (code F43.2), unlike DSM-IV-TR where is it is not classified under any particular group.

\section{Borders and boundaries}

The boundaries of adjustment disorder are not well defined in the current classifications. On the one hand, the distinction for normal adaptive reactions is not defined and, on the other, the distinction from other diagnoses such as mood and anxiety disorders is a matter of debate.

The distinction from normal adaptive reactions is not dealt with explicitly in either classification. Although ICD-10 specifies that social impairment should be present in order to make the diagnosis, there is no such requirement in DSM-IV-TR. This raises the possibility that by using DSM-IVTR the diagnosis could be applied in the face of proportionate and adaptive reactions to stressful events. This issue was raised when adjustment disorder was first introduced into DSM-I (Fabrega 1987). At that time, it was argued that it represented an attempt at medicalising problems of living. ICD-10 has to some extent recognised this danger and applied more rigorous criteria. DSM-IV-TR, on the other hand, requires that the symptoms should be clinically significant, although what this means is not specified.

\section{BOX 1 Features of adjustment disorder in ICD-10 and DSM-IV}

- The presence of a stressor is essential to making the diagnosis

- The onset of symptoms must proceed the stressor

- The symptoms begin within 1 month (ICD-10) or 3 months (DSM-IV) of the stressor

- The symptoms resolve within 6 months of termination of the stressor

- Symptoms may be prolonged beyond this if there are continuing consequences resulting from the stressor

- The diagnosis cannot be made in the presence of another mental state diagnosis

- The subtypes refer to the predominant symptoms (with depression, with anxiety, with disturbance of conduct, mixed types and other)

- Neither system of classification specifies diagnostic criteria in terms of symptom severity or numbers, apart from general principles, thus deviating from the approach used for other diagnoses

- The condition may be acute or chronic
Patricia Casey is Professor of Psychiatry at University College Dublin, and a consultant psychiatrist in the Mater Misericordiae University Hospital, Dublin, Ireland. Her research interests include suicidal behaviour and adjustment disorder. Faraz Jabbar is a senior registrar in learning disability at Stewarts Care, Palmerstown, Dublin, Ireland, and his research interest is in mood disorders.

Correspondence Patricia Casey, Professor of Psychiatry, Mater Misericordiae University Hospital, Eccles Street, Dublin 7, Ireland. Email: apsych@mater.ie 
On the other side of the adjustment disorder border lie the major diagnoses seen in psychiatric practice. The difficulty of distinguishing adjustment disorder from major depression in particular has been identified (Casey 2006), with few differences between the two in terms of symptom severity, personality disorder or social functioning. It has been suggested that the expansion in the prevalence of 'depression' in community-based epidemiological studies has come about because transient reactions are misdiagnosed and mopped up by the category of major depression (Regier 1998), a diagnosis that has expanded beyond clinical utility (Parker 2005).

The problem of distinguishing major depression from adjustment disorder using structured interviews should hardly be surprising. These instruments base the diagnosis on symptom numbers and duration and assess symptoms cross-sectionally, whereas adjustment disorder is a diagnosis that is inherently aetiological and longitudinal. So the conceptual frameworks of major depression and adjustment disorder differ significantly, yet both classifications deal with this simplistically by stating that a diagnosis of adjustment disorder cannot be made if the threshold for another diagnosis is reached, relegating adjustment disorder to subsyndromal status.

The distinction between adjustment disorder and minor depression, mild depression, subclinical depression or subsyndromal depression has not been formulated either and it is possible that these terms are used interchangeably.

Case vignette 1 considers the boundaries of adjustment disorder between normal reactions and major depression (Box 2).

\section{Epidemiology}

In population studies, adjustment disorder has not been considered in the National Comorbidity Survey Replication (Kessler 2005), National Psychiatric Morbidity Surveys (Jenkins 1997) or Epidemiologic Catchment Area Study (Myers 1984). By way of contrast, the Outcomes of Depression International Network (ODIN) did include adjustment disorder (Ayuso-Mateos 2001) and the prevalence varied between countries and by gender, with females in rural Finland having the highest prevalence (1.9\%) and other groups having a prevalence of less than $1.0 \%$ (e.g. males in urban Ireland). Maercker et al (2008) studied a representative sample of elderly persons from Switzerland (aged 65-96 years) and found a prevalence rate of $2.3 \%$ for adjustment disorder, compared with $2.3 \%$ for major depressive disorder and $0.7 \%$ for PTSD. Using a narrow definition
B0X 2 Case vignette 1

Ms $X$, aged 50, was diagnosed with Parkinson's disease. She was referred with low mood, tearfulness, poor concentration, hopelessness, loss of interest and insomnia since the diagnosis 1 month earlier. She was showing no response to treatment for her physical condition at the time of referral. She had no psychiatric history and had worked as a nurse up to the time of diagnosis - she is the only breadwinner. She has two teenage children and her husband was recently made redundant.

Comment

Given that Ms X is the only breadwinner, with two dependent children and insight into the prognosis, it could be argued that her distress response was proportionate and appropriate. On the other hand, her symptoms have lasted for 4 weeks and she meets the criteria for depressive episode. Alternatively, it could be argued that she has an adjustment disorder, given the close time relationship between the onset of symptoms following the neurological diagnosis, and the absence of any response to treatment.

of adjustment disorder, similar in symptoms to PTSD but triggered by day-to-day events rather than major traumas, Maercker et al (2012) found a prevalence of $0.9 \%$ and $1.4 \%$ with and without the impairment criteria respectively, in the general population.

Adjustment disorder is a notably common diagnosis in emergency department populations. Among individuals who undergo psychiatric assessment following self-harm, adjustment disorder was the clinical diagnosis in $31.8 \%$ and major depression in 19.5\% (Taggart 2006); however, when the Structured Clinical Interview for DSM-IV-TR disorders (SCID) was used, the figures changed, with adjustment disorder dropping to $7.8 \%$ and major depression increasing to $36.4 \%$. Brakoulias et al (2010) studied patients referred to a new psychiatric emergency care centre and found adjustment disorder to be the most common diagnosis, present in $35.9 \%$ of cases. Kropp et al (2007), using data on all individuals treated or assessed for mental illness in the emergency department over 1 year, diagnosed adjustment disorder in $6.7 \%$ of patients, after acute alcohol intoxication (20.2\%) and paranoid schizophrenia (14.2\%).

Adjustment disorder accounts for a significant proportion of referrals to consultation liaison psychiatry services in general hospitals and is the primary diagnosis in 12\% of cases (Strain 1998).

In Europe, too, adjustment disorder accounts for a significant proportion of psychiatric morbidity 
in hospitals: one study of 56 consultation liaison psychiatry services in 11 European countries found that although self-harm was the most common reason for seeking psychiatric assessment (17\%), adjustment disorder and PTSD accounted for $12.4 \%$ of referrals (Huyse 2001). Further studies in consultation liaison psychiatry indicate that adjustment disorder is almost three times as common as major depression in acutely ill medical in-patients $(13.7 \%$ as opposed to $5.1 \%)$ (Silverstone 1996) and almost twice as common as mood disorders in obstetric and gynaecological liaison services (Rigatelli 2002). The contribution of additional psychosocial and environmental stressors to adjustment disorder in this setting has also been recognised (Snyder 1990).

However, a changing pattern has been reported, with the diagnosis of adjustment disorder declining in tandem with an increase in the diagnosis of major depression (Diefenbacher 2002). This may reflect not so much a change in their prevalence as a change in the 'culture of diagnosis' (Strain 2008) stimulated by the availability of newer antidepressants for the treatment of major depression.

Yet the continuing salience of adjustment disorder is evident from a recent meta-analysis (Mitchell 2011). This identified adjustment disorder as the diagnosis in $15.4 \%$ and major depression in $16.5 \%$ of cases among studies conducted in palliative care settings, while in oncological and haematological settings adjustment disorder was the diagnosis in $19.4 \%$ of cases and major depression in $16.3 \%$ of cases.

Studies of diagnoses of individuals in contact with psychiatric services are scarce. Among intake assessments at a rural and an urban clinic, adjustment disorder was the most common clinical diagnosis, made in $36 \%$ of those seen, but this dropped to just over $11 \%$ when the SCID was used. Concordance between clinical and SCID diagnoses was lower for adjustment disorder than for any other diagnosis (Shear 2000). Among inpatients, adjustment disorder was the diagnosis in 9\% (Koran 2003).

Adjustment disorder is said to be very common in primary care, where family practitioners deal with the long-term impact of physical illness as well as the consequences of social and interpersonal problems. Prevalence rates of 11-18\% among those consulting with mental health problems have been described in older studies (Casey 1984; Blacker 1988). In a recent study, the SCID-I identified adjustment disorder in $2.8 \%$ of a population of primary care patients (Fernández 2012), but only 2 of the 110 cases identified were detected by the general practitioner.

\section{Psychobiology of adjustment disorder}

Biological studies are scarce but some are slowly emerging that show differences between adjustment disorder and major depression. In individuals expressing suicidal ideation, post-dexamethasone suppression of cortisol levels was negatively correlated with symptom scores only in those with a diagnosis of major depression; there was no correlation in those with adjustment disorder (Lindqvist 2008). Among people with adjustment disorder in the context of workplace bullying (Rocco 2007; Di Rosa 2009), dexamethasone suppression test and other aspects of the hypothalamus-pituitary-adrenal axis were found to be normal. Although limited in number and scope, these studies point to a distinction between major depression/depressive episode and adjustment disorder.

\section{Making the diagnosis}

\section{Structured interview}

It is generally assumed that structured diagnostic interviews are the gold standard for making diagnoses in epidemiological settings. It is unclear whether this is true for adjustment disorder, since it is not incorporated into many of the commonly used instruments, such as the Clinical Interview Schedule (CIS) (Lewis 1992) or the Composite International Diagnostic Interview (CIDI) (Kessler 2004). The Schedules for Clinical Assessment in Neuropsychiatry (SCAN) (Wing 1990) does include adjustment disorder in the section on inferences and attributions. However, this comes after the criteria for all other disorders have been completed and there are no specific questions to assist the interviewer in making the diagnosis. The SCID (First 1995) and the Mini-International Neuropsychiatric Interview (M.I.N.I.) (Sheehan 1998) both incorporate a section on adjustment disorder, but in both it is trumped by the presence of any other diagnosis.

This problem with structured interviews has been highlighted by a number of studies in clinical settings. These showed that when structured interviews are used, major depression is the predominant diagnosis, whereas in the same patients when clinical diagnosis is used, adjustment disorder is the more common (Shear 2000; Taggart 2006).

Attempts to develop a screening instrument for adjustment disorder based on the Hospital Anxiety and Depression Scale (HADS) (Akechi 2004) or on the 1-Question Interview and Impact Thermometer (Akizuki 2003) have been shown to measure a general dimension of low mood but 
not to distinguish adjustment disorder from major depression.

At this point is it arguable that clinical diagnosis which takes account of the context of symptoms and of the likely longitudinal course is superior to structured interviews when diagnosing adjustment disorder for research purposes.

\section{Clinical diagnosis}

\section{The individual}

Unexceptional events can trigger adjustment disorder, but events of a magnitude that in some people lead to PTSD, in others result in a cluster of symptoms best considered as adjustment disorders as they do not encapsulate the full PTSD spectrum.

When assessing an individual's reaction to a stressful event, it is important to take four key aspects into account, to help distinguish adjustment disorder from normal responses to stressors:

- the individual's personal circumstances and the context of the stressful event - for example, depending on their financial circumstances, an event such as redundancy might be devastating for one individual, but welcomed by another;

- the proportionality between the triggering event and symptom severity - for example, a minor event is unlikely to have a significant impact on a person with well-developed coping skills, whereas a vulnerable person could have a severe reaction;

- cultural and subcultural norms for emotional expression and emotional responses - for example, some cultures allow for the expression of emotion very openly and noticeably and such manifestations might be considered normal, whereas in others this might be regarded as indicating pathology;

- severity and duration of resultant functional and social impairment - for example, brief reactions to stressful events including functional impairment can occur in non-pathological reactions such as grief following a bereavement, but when the impairment persists the reaction might be considered abnormal.

ICD-10 (World Health Organization 1992) opines that personal vulnerability plays a greater role in adjustment disorder than in other psychiatric conditions. So, what is the evidence for this?

The frequency of personality disorder among individuals with adjustment disorder in comparison with those with other depressive disorders seems to be no different (Casey 2006), although studies are scarce. Other investigators have focused on personality dimensions, especially neuroticism (For-Wey 2006) and attachment style (For-Wey 2002). Using individuals chosen from the military with a diagnosis of adjustment disorder, neuroticism emerged as one of the dimensions predisposing to adjustment disorder (For-Wey 2006). Attachment style, maternal overprotection and paternal abuse were also identified as risk factors for later adjustment disorder (Giotakos 2002). However, no comparisons were made with patients who had other psychiatric disorders, so the relevance of these findings is unclear.

The presence of social supports has been seen as buffering the impact of adverse events in people with depressive disorders and, although not studied specifically in adjustment disorder, may be relevant in this condition also.

\section{The stressor}

The essential requirement for a diagnosis of adjustment disorder is that the symptoms must be triggered by a stressful event. The maximum time lag between the event and the onset of symptoms is 1 month in ICD-10 and 3 months in DSM-IV-TR. According to ICD-10:

'The stressor may have affected the integrity of an individual's social network (through bereavement or separation experiences) or the wider system of social supports and values (migration or refugee status). The stressor may involve only the individual or also his or her group or community' (World Health Organization 1992).

The type of event varies from those that are considered everyday, such as a row with a friend, to those that are more serious, such as being bullied in the workplace. A study comparing patients with major depression and those with adjustment disorder identified a higher proportion of events related to marital problems and fewer related to occupational or family stressors in the adjustment disorder group (Despland 1995), but as these are not specific to either diagnosis, they are unlikely to be diagnostically helpful. There may be multiple simultaneous stressors, which may further complicate the clinical picture. A relatively minor stressor, which appears to have little effect on its own, may have an additive effect on earlier, major stressors, and thus precipitate adjustment disorder.

Another key feature of adjustment disorder is that the symptoms resolve spontaneously after the stressor is removed. This feature may help separate adjustment disorder from other disorders, although this point of distinction requires a longitudinal perspective on the course of the symptoms. Clinically, it presents as mood reactivity. Experimentally, removing the person from the stressful environment might help 
clarify the diagnosis, as improvement is likely to be significant in adjustment disorder but more transient and superficial in individuals with major depression/depressive episode. Additionally, the closer the temporal proximity between stressor and symptoms, the more likely is the diagnosis to be one of adjustment disorder. Symptoms may recur when there is cognitive proximity to the stressor, such as when speaking about it (e.g. in the context of litigation).

\section{Symptoms and behaviour}

Adjustment disorder is generally regarded as a 'mild' condition, although the evidence for a distinction based on severity is ambiguous. One study in a general population sample (Casey 2006) failed to find any distinction in symptom severity or in social functioning between depressive episode and adjustment disorder with depression subtype. On the other hand, a recent study (Fernández 2012) identified some differences, notably better quality of life, in patients with adjustment disorder (depressed or anxious subtypes) compared with those diagnosed with major depression or generalised anxiety.

The absence of melancholic features might also be of assistance in distinguishing those with adjustment disorder from those with a depressive episode or major depression. Yates et al (2004) examined a group of patients with major depression with and without physical illness. Patients with physical illness were less likely to display melancholic features, raising the possibility that the greater the role of environmental factors, the less likely are the typical melancholic symptoms to be present. Since environmental factors are prominent in adjustment disorder, it is possible that these symptoms might help distinguish individuals with adjustment disorder from those with more biologically determined depression. Only further studies will demonstrate whether these symptoms have sufficient specificity. This has led to recommendations (Baumeister 2009) that this should be encapsulated in the revisions for DSM-5 and ICD-11.

Suicidal behaviour is common in people with adjustment disorder. As many as $25 \%$ of adolescents with a diagnosis of adjustment disorder engage in this behaviour (Pelkonen 2005), and this rises to 60\% among adults (Kryzhanovskaya 2001). Moreover, suicidal behaviour emerges several months earlier in patients with adjustment disorder in comparison with major depression (<1 v. 3 months respectively) (Runeson 1996).

Case vignette 2 illustrates adjustment disorder with depressed mood (Box 3).
B0X 3 Case vignette 2
Mr Y was a 45-year-old man admitted to a psychiatric unit having been rescued when he tried to drown himself. He had left a suicide note in his car. He reported increasingly low mood since his marriage broke up 6 months earlier. Shortly thereafter he had been made redundant. Although he had found another job, it was at a much reduced salary. This had resulted in mounting debt. When he moved out of his home, he had to rent a small flat in a run-down part of the city, this being all that he could afford. He was embarrassed to meet his friends and siblings because of his situation. As his accommodation was so poor, his evenings were spent in the pub, where he drank and ate, usually on his own. He returned home only to sleep. He experienced initial insomnia. He had no interest in his previous hobbies such as watching football and he saw no hope for the future. He continued to go to work and was satisfied that he was doing it well. Seeing no solution to his debts or accommodation problems, he decided to end his life.

During his stay in hospital he was given no medication other than hypnotics for the first two nights. He was eating well, attending all activities and engaging in conversation with staff and fellow patients. His mood improved within a few days and he reported relief that his life had been saved. He also felt happy to be away from his situation and to be receiving help.

He received visits from close friends and siblings, who made offers of accommodation which he accepted. He was referred to a free financial advice service for help in addressing his debts and to the local council for help with housing.
He began to discuss his problems more openly and was discharged 2 weeks post-admission. Throughout the 3-month follow-up he remained symptom-free and continued the process of resolving his debt and accommodation problems. He did not require medication at any point up to his discharge to his general practitioner some 3 months after his admission.

Comment

Mr Y's history showed that the onset of symptoms was closely related in time to the break-up of his relationship and was maintained and eventually worsened by a number of factors, including financial difficulties and housing problems. In addition, he was socially isolated and so had no support from those who might have been able to help him, either emotionally or practically. Although his mood was low, he continued to work. His use of alcohol may have contributed to his low mood. His increasing hopelessness and sense of helplessness led him to make a serious suicide attempt. Once he was in an environment that removed him from his adverse circumstances and offered practical assistance and emotional support, his symptoms resolved rapidly with minimal pharmacotherapy

This case vignette indicates that adjustment disorder can persist while adverse social circumstances continue and that it can in some cases be associated with serious suicidal behaviour. This history illustrates the possible conflation of adjustment disorder with depressive episode, since $\mathrm{Mr} \mathrm{Y}$ might easily have been prescribed antidepressants, when in fact his symptoms were driven exclusively by his circumstances.

\section{Differential diagnosis}

The differential diagnoses to be considered in people with adjustment disorder are listed in Box 4.

The differential diagnosis includes the common differentials for anybody with either depressive or anxiety symptoms. The closeness in time between the occurrence of the stressor and the onset of symptoms is very useful in deciding between adjustment disorder and either a depressive episode or generalised anxiety disorder. The fact that there is cognitive proximity between the symptoms and the stressor (e.g. talking about the event worsens the symptoms, while at other times the individual 
BOX 4 Differential diagnosis in adjustment disorder

- Normal reaction to stress

- Major depression (or other symptom-related disorder e.g. generalised anxiety disorder)

- Major depression (or other symptom-related disorder e.g. generalised anxiety disorder) in evolution

- Emotionally unstable personality disorder

- PTSD and acute stress reaction

- Substance misuse

- Dysthymia

is symptom-free) is more suggestive of adjustment disorder rather than other Axis I disorders.

Emotionally unstable personality disorder is included because shifts in mood can occur in response to stressful events. In this context, the transient changes that occur should not be labelled as adjustment disorder since these are inherent in emotionally unstable personality disorder. With regard to PTSD, events that are of such magnitude as to cause PTSD can also lead to adjustment disorder and this may be the more appropriate diagnosis when the full criteria for PTSD are absent. Shifts in mood can occur in individuals who misuse substances, particularly alcohol, and these should not be labelled as adjustment disorder unless there are co-occurring events driving the mood changes.

The possibility that the symptoms constitute a subthreshold condition such as mild or subclinical depression must also be considered. A problem, however, is that these are poorly defined and delineated, and apart from the requirement that adjustment disorder is preceded by a stressor, there is nothing in the scientific literature to assist the clinician in differentiating between these. Mild depression is recognised in ICD-10, but apart from the requirement that adjustment disorder has a triggering stressor and that the disorder is expected to resolve when the stressor is removed, there is nothing additional to assist in deciding on one disorder or the other. Similar problems arise with respect to subsyndromal and subclinical depression, terms that seem to have arisen without any clarity as to their meaning (Snaith 1987). There is a case to be made for either defining or abandoning such terms.

When both the stressor and the symptoms persist, it is possible that a diagnosis of dysthymia will be made. However, a history of fluctuations in response to changes in the stressor will tend towards a diagnosis of chronic adjustment disorder. Changes in symptom severity that are independent of the stressor will point in the direction of dysthymia (with associated double depression). The patient's history may also be illuminating, in that those with adjustment disorder usually have no related history, whereas those with dysthymia have a history of persistent low mood often lasting many years and beginning usually in early adulthood.

\section{Evidence-based management}

There is very little to assist the clinician in making treatment decisions for adjustment disorder, since randomised controlled trials are scarce. No guidelines from the National Institute for Health and Clinical Excellence exist either. Indeed, it is questionable whether specific interventions are even required, since generally the symptoms resolve spontaneously, unless there are continuing stressors or reminders that maintain them.

Insofar as treatment is required, brief psychological treatments are the preferred option (Strain 2008). This seems appropriate, given the usually time-limited nature of the disorder. There are few randomised controlled trials and an array of psychological and pharmacological interventions have been tried with some success, both individually and in groups and underpinned by different theoretical models (Casey 2009).

Pharmacological treatments have a limited role apart from symptomatic treatment of anxiety and insomnia with benzodiazepines (Shaner 2000). However, a number of placebo-controlled trials of herbal remedies have been evaluated, with kava kava (Voltz 1997) and valerian plus other extracts (Bourin 1994) showing some benefit in adjustment disorder with anxiety symptoms.

There have been no randomised controlled trials of antidepressants for adjustment disorder with depression, yet they are frequently prescribed for this condition: a recent study revealed that $45 \%$ of patients with a diagnosis of adjustment disorder received an antidepressant (Fernández 2012). In the USA, antidepressants have been increasingly prescribed for psychiatric disorders: between 1996 and 2005 the most marked increase in their use was for adjustment disorder, rising from $22 \%$ to 40\% (Olfson 2009).

Finally, one study comparing antidepressants, placebo, supportive psychotherapy and benzodiazepines found that all were associated with significant improvement (de Leo 1989), lending further credence to the possibility that no specific intervention may be required. 


\section{Outcome and prognosis}

There is very little information on the diagnostic stability of adjustment disorder in comparison with other diagnoses. For example, does adjustment disorder augur some more sinister diagnosis or does it recur, albeit retaining the same taxonomic diagnosis? In study reported that the diagnostic stability of the ICD-10 group within which adjustment disorder is classified (neurotic and stress-related disorders) was much lower, at $37 \%$, than the $73 \%$ stability achieved for depressive disorders (Daradkeh 1997). However, numbers were small and adjustment disorder was not examined separately. Another study found that adjustment and anxiety disorders were the least stable, at around 34\%, but again adjustment disorder was not examined on its own (Huquelet 2001). Greenberg et al (1995), a vociferous critic of the diagnosis, reported that $59 \%$ of patients with an admission diagnosis of adjustment disorder were re-diagnosed as having a primary diagnosis of substance misuse. Owing to the paucity of information, it is not possible to describe the frequency or direction of diagnostic change in those with an initial diagnosis of adjustment disorder in comparison with other categories.

By contrast, a definite point of departure from other common mental disorders is the better prognosis in people with adjustment disorder. Not only does symptom improvement occur more quickly, but readmission rates are lower than in other diagnostic groups sharing the same symptoms (Jones 2002), most likely because of the temporal character of the stressor. Whether a diagnosis of adjustment disorder places the person at risk of some other disorder has not been examined, but the answer is likely to be negative, since this is a condition that is exclusively stressorinduced and, as already mentioned, it has a lower readmission rate.

However, adjustment disorder is neither benign nor mild as some suggest. It is the diagnosis in up to a third of young people who die by suicide (Lönnqvist 1995) and among all suicide deaths in low- and middle-income countries it is the most common diagnosis (Manoranjitham 2010).

\section{ICD-11 and DSM-5}

A major concern for those framing ICD11 and DSM-5 is how and where to include adjustment disorder in these new classifications. It is recommended that all the trauma-related disorders, including adjustment disorder, should move from the anxiety disorder section in DSM-5 to a new section incorporating disorders that are stress related (Freidman 2011), in recognition of the common aetiology of some disorders rather than their common phenomenology. This would align ICD and DSM.

Of further relevance is whether adjustment disorder should continue to be a subsyndrome that cannot be diagnosed when the threshold for another disorder is reached. Acceptance of adjustment disorder as a full-blown disorder would inevitably lead to greater clarity in its diagnosis and would stimulate research into its many facets. For this to happen, adjustment disorder must be accorded specific diagnostic criteria, as detailed by some (Baumeister 2009).

Screening and diagnostic tools that can be used in research in adjustment disorder need to be developed. These must not only distinguish adjustment disorder from normal adaptive reactions but must also separate adjustment disorder from other possible diagnoses. However, such developments are unlikely to occur until adjustment disorder becomes a fully accepted syndrome in its own right. It remains to be seen whether radical steps such as these are considered for the forthcoming editions of ICD and DSM.

\section{Conclusions}

Adjustment disorder is an underutilised diagnosis in clinical practice. It lacks any well-developed criteria in the current classifications and is poorly conceptualised. For these reasons it is underresearched. It is conflated with other Axis I diagnoses and many of the treatments offered are not evidence based. The development of ICD-11 and DSM-5 will be an opportunity to establish better diagnostic criteria for this condition, thus enabling research to consider the myriad questions yet to be answered.

\section{References}

Akechi N, Okuyama T, Sugawara Y, et al (2004) Adjustment disorders and post-traumatic stress disorder in terminally ill cancer patients: associated and predictive factors. Journal of Clinical Oncology 22: 1957-65.

Akizuki N, Akechi T (2003) Developing a brief screening interview for adjustment disorder and major depression in patients with cancer. Cancer 97: 2605-13.

American Psychiatric Association (1980) Diagnostic and Statistical Manual of Mental Disorders (3rd edn) (DSM-III). APA.

American Psychiatric Association (2000) Diagnostic and Statistical Manual of Mental Disorders (4th edn, text revision) (DSM-IV-TR). APA.

Ayuso-Mateos JL, Vázquez-Barquero JL, Dowrick C, et al (2001) Depressive disorders in Europe: prevalence figures from the ODIN study. British Journal of Psychiatry 179: 308-16.

Baumeister H, Maercker A, Casey P (2009) Adjustment disorder with depressed mood: a critique of its DSM-IV and ICD-10 conceptualizations and recommendations for the future. Psychopathology 42 : 139-47. 


\section{MCO answers \\ 1 a $\quad 2$ e $\quad 3$ e $\quad 4$ a $\quad 5 d$}

Blacker CVR, Clare AW (1988) The prevalence and treatment of depression in general practice. Psychopharmacology 95 (suppl): S14-27.

Bourin M, Bougerol T, Guitton B, et al (1994) A combination of plant extracts in the treatment of outpatients with adjustment disorder and anxious mood: controlled study versus placebo. Fundamentals of Clinical Pharmacology 11: 127-32.

Brakoulias V, Mandali R, Seymour J, et al (2010) Characteristics of admissions to a recently opened psychiatric emergency care centre. Australasian Psychiatry 18: 326-9.

Casey PR, Dillon S, Tyrer P (1984) The diagnostic status of patients with conspicuous psychiatric morbidity in primary care. Psychological Medicine 14: 673-81.

Casey P, Maracy M, Kelly BD, et al (2006) Can adjustment disorder and depressive disorder be distinguished? Results from ODIN. Journal of Affective Disorders 92: 291-7.

Casey P (2009) Adjustment disorder: epidemiology, diagnosis and treatment. CNS Drugs 23: 927-38.

Daradkeh T, El-Rufaie 0, Younis Y, et al (1997) The diagnostic stability of ICD-10 psychiatric diagnoses in clinical practice. European Psychiatry 12: $136-9$.

de Leo D (1989) Treatment of adjustment disorders: a comparative evaluation. Psychological Reports 64: 51-4.

Despland JN, Monod L, Ferrero F (1995) Clinical relevance of adjustment disorder in DSM-III-R and DSM-IV. Comprehensive Psychiatry 36: 454-60.

Di Rosa AE, Gangemi S, Cristani M, et al (2009) Serum levels of carbonylated and nitrosylated proteins in mobbing victims with workplace adjustment disorders. Biological Psychology 82: 308-11.

Diefenbacher A, Strain JJ (2002) Consultation-liaison psychiatry: stability and change over a 10-year period. General Hospital Psychiatry 24: 24956.

Fabrega H, Mezzich J (1987) Adjustment disorder and psychiatric practice: cultural and historical aspects. Psychiatry 50: 31-49.

Fernández A, Mendive JM, Salvador-Carulla L, et al (2012) Adjustment disorders in primary care: prevalence, recognition and use of services. British Journal of Psychiatry 201: 137-42.

First MB, Spitzer RL, Williams JW, et al (1995) Structured Clinical Interview for DSM-IV (SCID 1). New York Biometric Research Department, New York State Psychiatric Institute.

For-Wey L, Fin-Yen L, Bih-Ching S (2002) The relationship between life adjustment and parental bonding in military personnel with adjustment disorder in Taiwan. Military Medicine 167: 678-82.

For-Wey L, Fin-Yen L, Bih-Ching S (2006) The pre-morbid personality in military students with adjustment disorder. Military Psychology 18: 77-88.

Freidman MJ, Resick PA, Bryant R, et al (2011) Classification of trauma and stressor-related disorders in DSM-5. Depression and Anxiety 28: 737-49

Giotakos 0, Konstantakopoulos G (2002) Parenting received in childhood and early separation anxiety in male conscripts with adjustment disorder. Military Medicine 167: 28-33.

Greenberg WM, Rosenfeld D, Ortega E (1995) Adjustment disorder as an admission diagnosis. American Journal of Psychiatry 152: 459-61.

Huquelet P, Schneider El, Gueddari N, et al (2001) Stability of DSM-III-R diagnoses: study of a case register. Psychopathology 34: 118-22.

Huyse F, Herzog T, Lobo A, et al (2001) Consultation-liaison psychiatric service delivery: results from a European study. General Hospital Psychiatry 23: 124-32.

Jenkins R, Lewis G, Bebbington P, et al (1997) The National Psychiatric Morbidity surveys of Great Britain - initial findings from the household survey. Psychological Medicine 27: 775-89.

Jones R, Yates WR, Zhou MH (2002) Readmission rates for adjustment disorders: comparison with other mood disorders. Journal of Affective Disorders 71: 199-203.

Kessler RC, Ustun TB (2004) The World Mental Health (WMH) survey initiative Version of the World Health Organisation (WHO) Composite
International Diagnostic Interview (CIDI). International Journal of Methods in Psychiatric Research 13: 93-121.

Kessler RC, Chiu WT, Demler 0, et al (2005) Prevalence, severity, and comorbidity of 12-month DSM-IV disorders in the National Comorbidity Survey Replication. Archives of General Psychiatry 62: 617-27.

Koran LM, Sheline Y, Imai K, et al (2003) Medical disorders among patients admitted to a public sector psychiatric in-patient unit. Psychiatric Services 53: 1623-5.

Kropp S, Andreis C, te Wildt B, et al (2007) Characteristics of psychiatric patients in the accident and emergency department (ED). Psychiatrische Praxis 34: $72-5$

Kryzhanovskaya L, Canterbury R (2001) Suicidal behaviour in patients with adjustment disorders. Crisis 22: 125-31.

Lewis G, Pelosi AJ, Araya R, et al (1992) Measuring psychiatric disorders in the community: a standardised assessment for use by lay interviewers. Psychological Medicine 22: 465-86.

Lindqvist D, Träskman-Bendzl L, Vang F (2008) Suicidal intent and the HPA axis characteristics of suicide attempters with major depression and adjustment disorders. Archives of Suicide Research 12: 197-207.

Lönnqvist JK, Henricksson MM, Sisometsä ET, et al (1995) Mental disorders and suicide prevention. Psychiatry and Clinical Neuroscience 49 (suppl 1): S111-6.

Maercker A, Forstmeier S, Enzler A, et al (2008) Adjustment disorders, posttraumatic disorder, and depressive disorders in old age: findings from a community survey. Comprehensive Psychiatry 49: 113-20.

Maercker A, Forstmeier S, Pielmaier L, et al (2012) Adjustment disorders: prevalence in a representative nationwide survey in Germany. Social Psychiatry and Psychiatric Epidemiology 47: 1745-52.

Manoranjitham SD, Rajkumar AP, Thangadurai P, et al (2010) Risk factors for suicide in rural south India. British Journal of Psychiatry 196: 26-30.

Mitchell AJ, Chan M, Bhatti H, et al (2011) Prevalence of depression, anxiety and adjustment disorder in oncological, haematological and palliative care settings: a meta-analysis of 94 interview based studies. Lancet Oncology 12: 160-74.

Myers JK, Weissman MM, Dischler GL, et al (1984) Six-month prevalence of psychiatric disorders in three communities 1980 to 1982. Archives of General Psychiatry 41: 959-67.

Olfson M, Marcus SC (2009) National patterns in antidepressant medication treatment. Archives of General Psychiatry 66: 848-56.

Parker G (2005) Beyond major depression. Psychological Medicine 35: $367-74$

Pelkonen M, Marttunen M, Henriksson M, et al (2005) Suicidality in adjustment disorder, clinical characteristics of adolescent outpatients. European Child and Adolescent Psychiatry 14: 174-80

Regier DA, Kaelber CT, Rae DS, et al (1998) Limitations of diagnostic criteria and assessment instruments for mental disorders: implications for research policy. Archives of General Psychiatry 55: 109-15.

Rigatelli M, Galeazzi GM, Palmieri G (2002) Consultation-liaison psychiatry in obstetrics and gynaecology. Journal of Psychosomatics, Obstetrics and Gynaecology 23: 165-72.

Rocco A, Martocchia A, Frugoni P, et al (2007) Inverse correlation between morning cortisol levels and MMPI psychaesthenia and depression scale scores in victims of mobbing with adjustment disorders. Neurology and Endocrinology 28: 610-3.

Runeson BS, Beskow J, Waern M (1996) The suicidal process in suicides among young people. Acta Psychiatrica Scandinavica 93: 35-42.

Shaner R (2000) Benzodiazepines in psychiatric emergency settings. Psychiatric Annals 30: 268-75.

Shear KM, Greeno C, Kang J, et al (2000) Diagnosis of non-psychotic patients in community clinics. American Journal of Psychiatry 157: 581-7.

Sheehan D, Lecrubier Y, Sheehan KH, et al (1998) The Mini-International Neuropsychiatric Interview (M.I.N.I.): the development and validation of a structured diagnostic psychiatric interview for DSM-IV and ICD-10. Journal of Clinical Psychiatry 59 (suppl 20): 22-33. 
Silverstone PH (1996) Prevalence of psychiatric disorders in medical inpatients. Journal of Nervous and Mental Disease 184: 43-51.

Snaith RP (1987) The concepts of mild depression. British Journal of Psychiatry 150: 387-93.

Snyder S, Strain JJ, Wolf D (1990) Differentiating major depression from adjustment disorder with depressed mood in a medical setting. General Hospital Psychiatry 12: 159-65.

Strain JJ, Smith GC, Hammer JS (1998) Adjustment disorder: a multisite study of its utilisation and interventions in the consultation-liaison psychiatry setting. General Hospital Psychiatry 20: 139-49.

Strain J, Diefenbacher A (2008) The adjustments disorders: the conundrums of the diagnoses. Comprehensive Psychiatry 49: 121-30.

Taggart C, O'Grady J, Stevenson M, et al (2006) Accuracy of diagnosis and routine psychiatric assessment in patients presenting to an accident and emergency department. General Hospital Psychiatry 8: 330-5.
Voltz HP, Kieser M (1997) Kava-kava extract WS 1490 versus placebo in anxiety disorders: a randomised placebo-controlled 25 week outpatient trial. Pharmacopsychiatry 30: 1-5.

Wing JK, Babor T, Brugha T, et al (1990) SCAN: Schedules for Clinical Assessment in Neuropsychiatry. Archives of General Psychiatry 47: 589-93.

World Health Organization (1978) The ICD-9 Classification of Mental and Behavioural Disorders: Clinical Description and Diagnostic Guidelines. WHO.

World Health Organization (1992) The ICD-10 Classification of Mental and Behavioural Disorders: Clinical Description and Diagnostic Guidelines. WHO.

Yates WR, Mitchell J, Rush AJ, et al (2004) Clinical features of depressed out-patients with and without co-occurring medical conditions in STAR*D. General Hospital Psychiatry 26: 421-9.

\section{MCOs}

Select the single best option for each question stem

\section{A diagnosis of adjustment disorder cannot} be made unless:

a a stressor is identified

b the individual is vulnerable

c the individual has a personality disorder

$\mathrm{d}$ the symptoms persist beyond 6 months

e marked anxiety is present.

\section{Adjustment disorder and major} depression can be distinguished from each other by the presence of the following symptoms:

a insomnia

b low mood

c poor concentration

\section{d suicidal ideation}

e none of the above.

3 Adjustment disorder is best treated with: a antidepressants

b psychodynamic psychotherapy

c interpersonal therapy

d any of the above

e none of the above.

4 Adjustment disorder may be diagnosed using the following interview schedule:

a SCID

b CIDI

c CIS

d Hospital Anxiety and Depression Scale

e Hamilton Rating Scale for Depression.

\section{The following controversies concerning} adjustment disorder have been resolved:

a the distinction from normal stress reactions

$\mathrm{b}$ the distinction from major depression

c their categorisation as a trauma-based diagnosis in DSM-5

$\mathrm{d}$ the good prognosis in most circumstances

e their diagnosis by an interview schedule. 